\title{
Hypophysitis Secondary to a Ruptured Rathke Cleft Cyst
}

\author{
Nancy McLaughlin, François Lavigne, Shaun Kilty, France Berthelet, Michel W. Bojanowski
}

Can. J. Neurol. Sci. 2010; 37: 402-405

Inflammatory lesions of the pituitary gland are rare especially when due to a ruptured Rathke cleft cyst (RCC). We present two cases of symptomatic hypophysitis secondary to ruptured RCC treated by fenestration and aspiration of the sellar content. We reviewed the English literature in order to assess the best surgical strategy to prevent recurrence.

\section{CASE Report}

\section{Case 1}

A 31-year-old female consulted for polydipsia and polyuria ongoing since a few weeks. She reported weight gain over the last eight months and amenorhea with galactorhea for the last four months. Physical examination revealed no abnormalities other than a decreased visual acuity in the left eye already noticed by the patient over the past three months. Biochemical and hematological data were within normal range. Endocrine work-up showed slightly increased prolactine, decreased luteinizing hormone/follicle stimulating hormone (LH/FHS), decreased free T4 and compensated insipidus diabetes. Magnetic resonance imaging (MRI) demonstrated a sellar cystic lesion with suprasellar extension, displacing the optic chiasm upwardly. The lesion was iso-intense on T1WI and slightly hyperintense on T2WI (Figure 1). The lesion's rim enhanced following gadolinium injection. The stalk did not enhance. The preoperative diagnosis was a non-secreting cystic macroadenoma.

The lesion was approached by an endoscopic endonasal transsphenoidal route. After opening a thin capsule beneath the dura, a whitish viscous substance spontaneously leaked out of the sella (Figure 2). After the mucoid substance was completely aspirated, the angled endoscope enabled a view inside the cavity and removal of free floating fragments of the capsule, sparing the pituitary gland. Intra-operative pathological findings were suggestive of an inflammatory process. In light of this information, the cavity was irrigated and surgery terminated.

Post-operatively, the patient fully recovered her visual field defect. Currently two years following surgery, the patient remains on replacement treatment. Her follow-up MRI showed no lesion recurrence.

The definitive pathological assessment showed fragments of pituitary tissue, with residual acini among tissue infiltrated with $\mathrm{T}$ and $\mathrm{B}$ lymphocytes and plasmocytes. A few giant cells were identified in the specimen. One of the fragments was lined by squamous cells, suggestive of a ruptured RCC with secondary hypophysitis (Figure 3). Special stains for micro-organisms yielded negative findings.

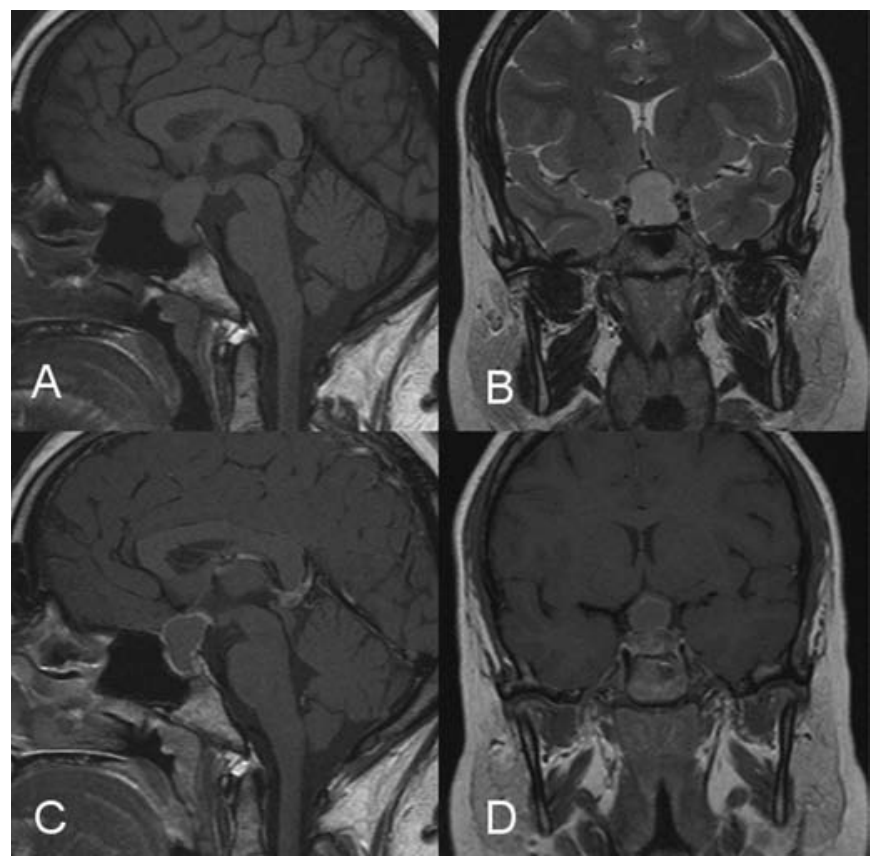

Figure 1: Magnetic resonance imaging in sagittal T1WI (A), coronal T2WI (B), sagittal (C) and coronal (D) T1WI with gadolinium. A sellar and suprasellar cystic mass is imaged, isointense on TIWI, slightly hyperintense on T2WI, and ring enhancing after gadolinium injection.

\section{Case 2}

A 28-year-old female was referred by her gynecologist for persistent postpartum amenorhea since 2001. Since 2004, she gained weight progressively and reported decreased libido. Physical examination, including ophthalmologic assessment, was normal. Biochemistry, hematology and endocrine work-up showed decreased LH, FSH, T4 and a slight decrease in insulin

From the Division of Neurosurgery (NM, MWB), Division of ENT Surgery (FL, SK), Department of Surgery, Department of Pathology (FB), Centre Hospitalier de l'Université de Montréal - Hôpital Notre-Dame, Montreal, Quebec, Canada.

Received September 24, 2009. Final Revisions Submitted December 15, 2009. Correspondence to: Michel W. Bojanowski, Neurosurgery Division, CHUM-Hôpital Notre-Dame, 1560 Sherbrooke St. East, Montreal, Quebec, H2L 4M1, Canada. 


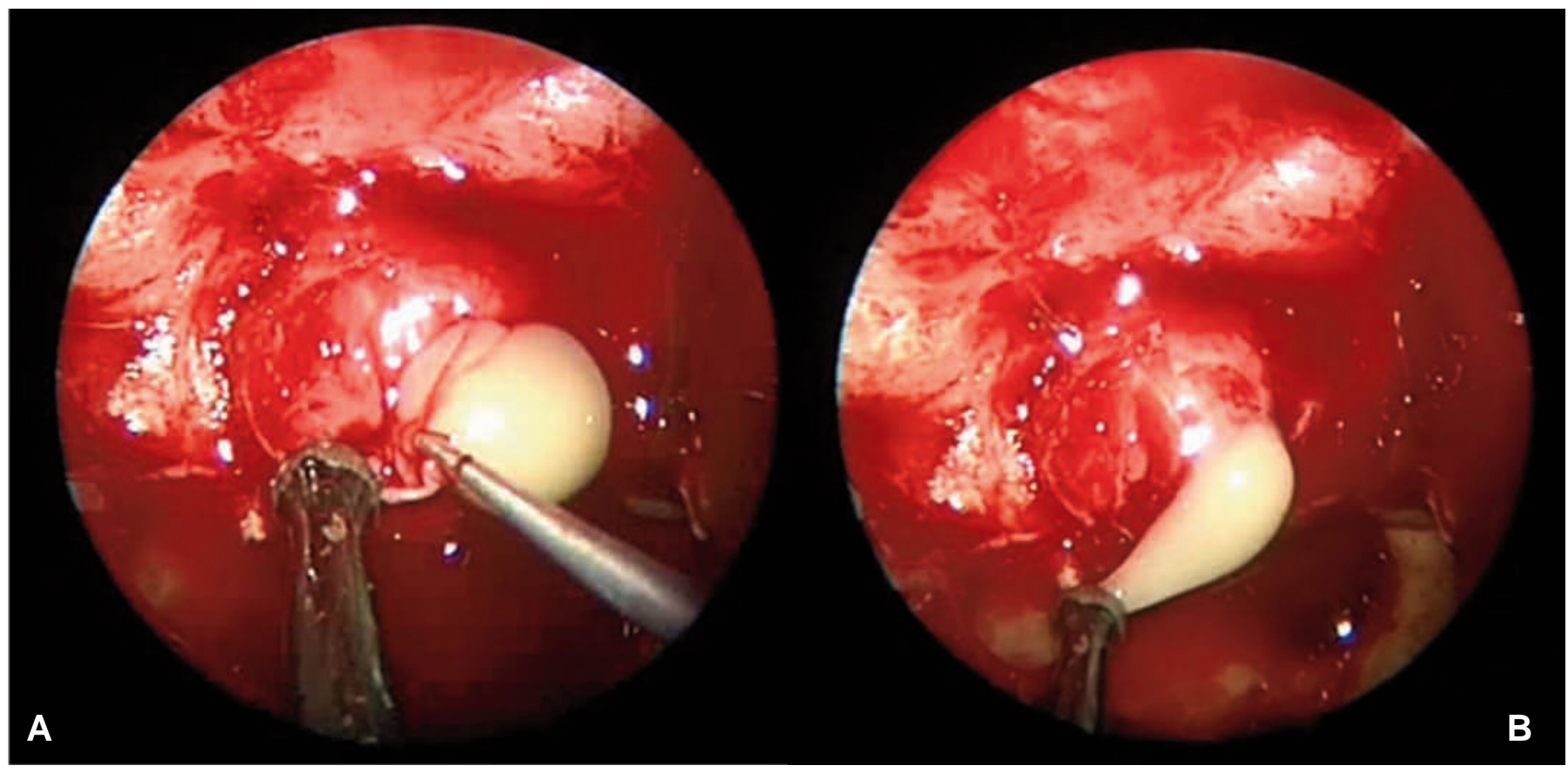

Figure 2: Intraoperative view. A) Through an endoscopic endonasal transphenoidal approach, the sella was reached. B) After incision of the dura, a whitish creamy fluid flowed from the cyst.

growth factor (IGF)-1. An MRI documented a sellar mass with suprasellar extension, displacing the optic chiasm upwardly. The lesion was iso-intense on T1WI and iso-intense on T2WI. Unfortunately, T1 sequences following gadolinium injection

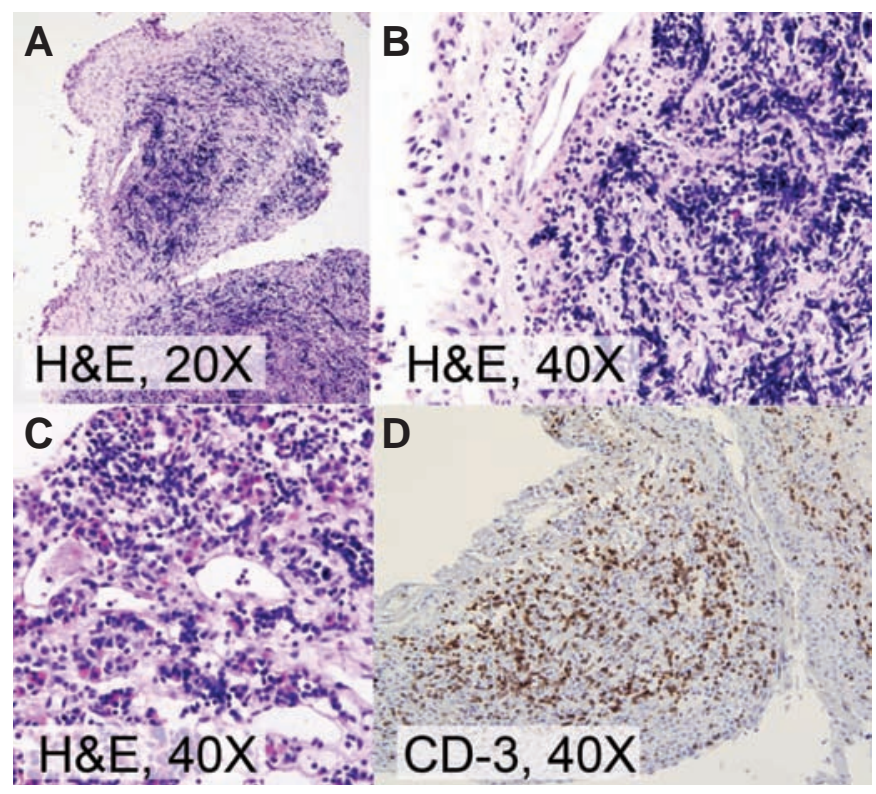

Figure 3: Pathology examination. H\&E stain $(A, B, C)$ and $C D-3$ immunostain (D). An inflammatory infiltrate largely made of lymphocytes and plasma cells disrupted the pituitary acini. A ciliated simple epithelium with focal squamous metaplasia covered the inflammed pituitary tissue. were unavailable preoperatively. Diagnosis prior to surgery was a non-secreting pituitary macroadenoma.

The lesion was approached by an endoscopic endonasal transsphenoidal route. Again, a whitish thick fluid poured out of the sella after incising a capsule (Figure 4). After the substance was completely evacuated, the cavity was inspected. Through the thin capsule, the gland was seen displaced to the right. Intraoperative pathology examination was inconclusive. The cavity was irrigated and the surgery terminated.

Post-operatively, the gonadotrope and growth hormone deficit recovered partially and the thyreotrophe deficit normalized. There was no clinical or radiological recurrence at 15 months follow-up.

The definitive pathology assessment revealed pituitary gland infiltrated by $\mathrm{T}$ and $\mathrm{B}$ lymphocytes as well as plasmocytes. In addition, numerous spumous macrophages and giant cells were present, consistent with a granulomatous inflammatory reaction. Some tissue fragments were lined by a cubic epithelium with areas of hyperplasia and metaplasia, suggestive of a ruptured RCC with secondary hypophysitis (Figure 5). Special stains did not identify any infectious organisms.

\section{Discussion}

Hypophysitis are rare sellar lesions that may be primary or secondary. Secondary hypophysitis have been described in association with systemic diseases such as sarcoidosis and Wegener granulomatosis or triggered by infectious agents (tuberculosis) or neighboring lesions such as adenomas, lymphomas or germinomas ${ }^{1}$. In rare circumstances, an hypophysitis may be secondary to ruptured RCC. No clinical presentation is pathognomonic of hypophysitis, most signs and 


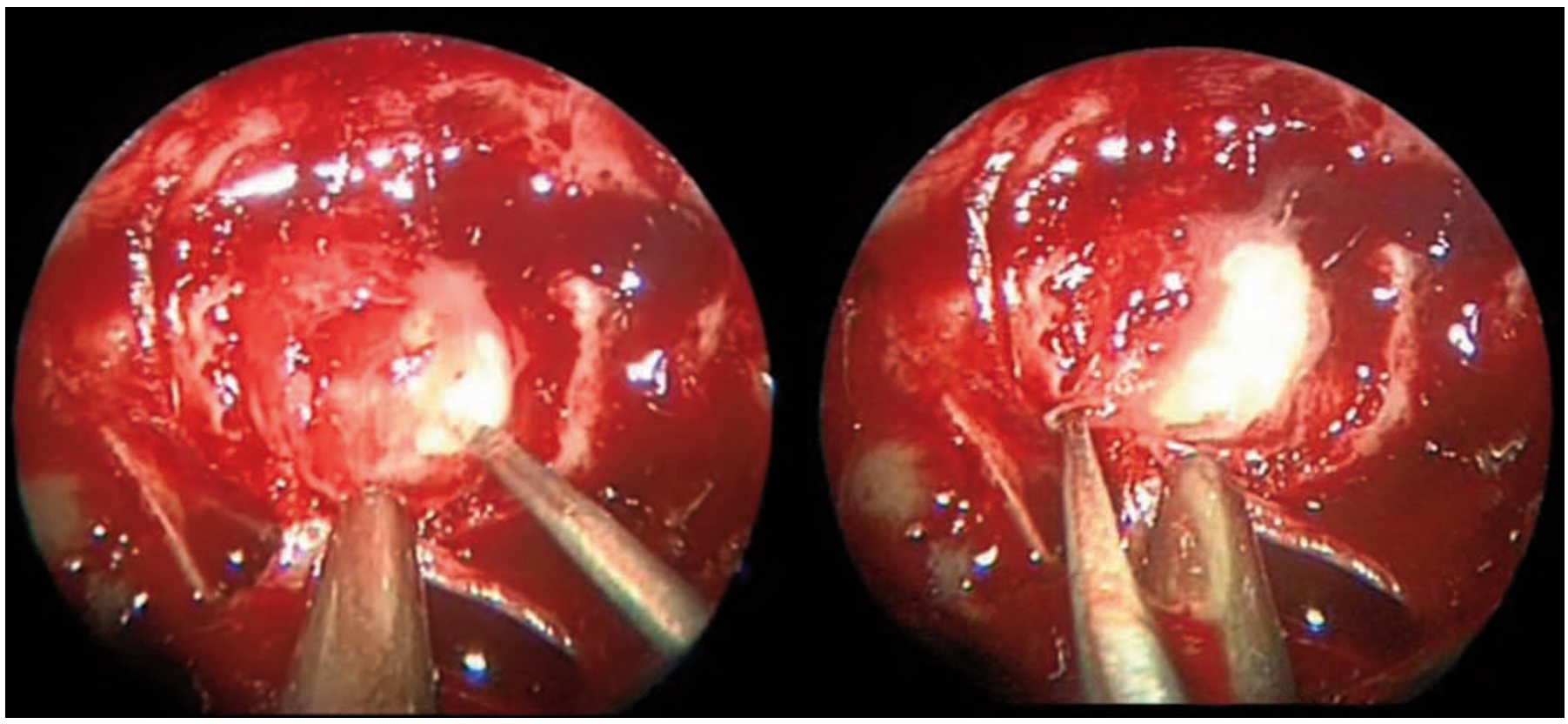

Figure 4: Intraoperative view. Through an endoscopic endonasal transphenoidal approach, the sella was reached. After incision of the dura, a whitish creamy fluid flowed from the cyst.

symptoms are related to the mass effect, namely headaches and visual deficits ${ }^{1,2}$.

The endocrine work-up may be normal, or may show hyperprolactinemia secondary to compression or infiltration of the pituitary stalk, or may present various levels of hormonal deficiency as a result of edema or destruction of pituitary tissue ${ }^{1}$. Insipidus diabetes may be causally related to neuro-hypophysis and/or stalk involvement.
Because hypophysitis and RCC show variable intensities on MRI, the diagnosis is difficult to make on an imaging basis. Magnetic resonance imaging features suggestive of hypophysitis include contrast enhancement of the mass and sparing cystic and necrotic parts, enlargement and hyperintensity on T2WI of the pituitary stalk and thickening of the parasellar dura and/or the sphenoid sinus mucosa $a^{1-3}$. However, given the numerous etiologies underlying secondary hypophysitis, no general

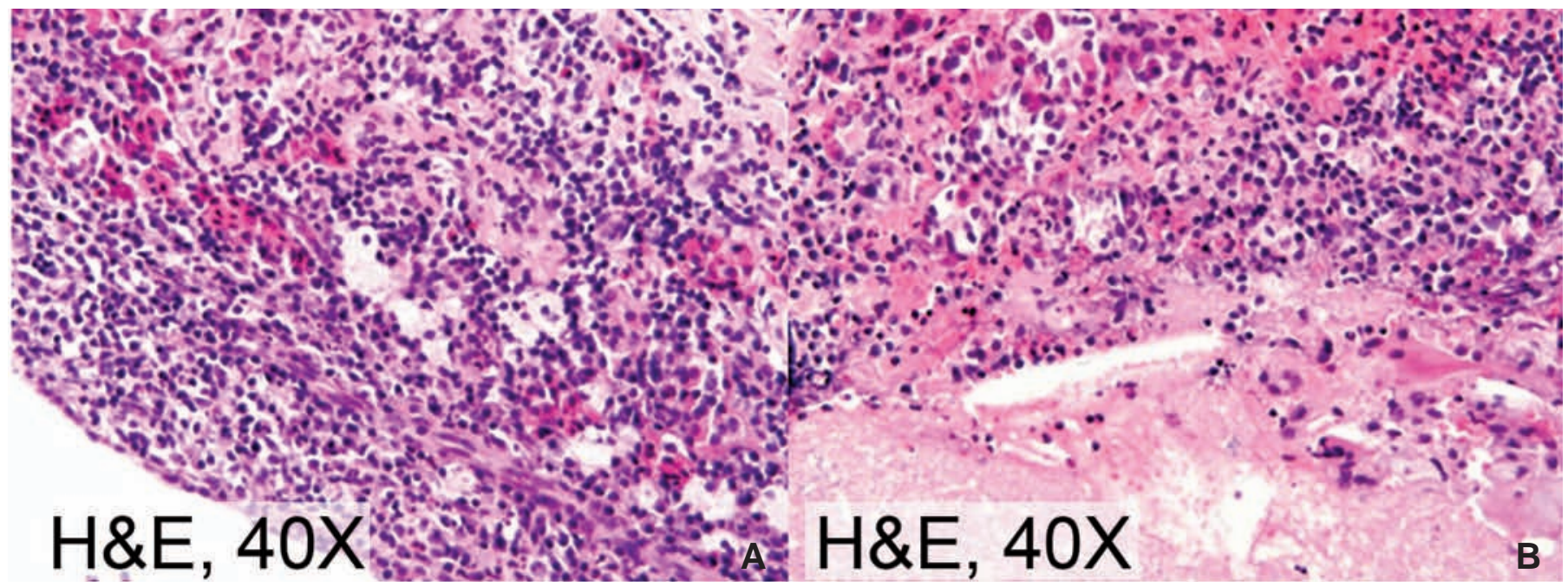

Figure 5: Pathology examination. $H \& E$ stain $(A \& B)$. A) Lymphocytes, plasma cells and multinucleated giant cells indicated a chronic granulomatous reaction. A simple epithelium with ciliated cells covered the pituitary tissue. B) Necrosis with cholesterol clefts (B) surrounded by inflamed pituitary tissue. 
correlation has been made regarding pre-operative imaging characteristics and intra-operative findings.

In addition to the present two patients, 14 other cases of hypophysitis with detailed descriptions of lymphocytic and/or granulomatous components have been reported secondary to a ruptured $\mathrm{RCC}^{1-12}$. Including our cases, 15 were treated surgically of which 14 had detailed surgical interventions. Twelve cases were treated by drainage of cyst and/or removal of more solid abnormal tissue. Gland removal was performed in one case given the difficulty of differentiating between normal gland and abnormal tissue ${ }^{7}$ and in another case without specification ${ }^{8}$. Of those treated with fenestration and aspiration of the abnormal sellar content, most patients remained on hormonal treatment following the surgery, to replace pre-operative hormonal deficit. However, two patients had improved endocrinologic work-up following such intervention ${ }^{9}$. Reported follow-up varies from 11 to 90 months. Conservative surgical treatment has resulted in good long term follow-up, with no reported recurrence.

In summary, neurosurgeons should suspect an inflammatory lesion when facing leakage of a thick whitish fluid from the sella. Intra-operative pathology examination is recommended to orient toward a conservative surgical treatment to minimize pituitary injury. As for other secondary hypophysitis, conservative surgical treatment of hypophysitis secondary to RCC, consisting of fenestration and aspiration of the sellar content, is appropriate to prevent recurrence.

\section{REFERENCES}

1. Flanagan DE, Ibrahim AE, Ellison DW, Armitage M, Gawne-Cain M, Lees PD. Inflammatory hypophysitis - the spectrum of disease. Acta Neurochir (Wien). 2002;144:47-56.

2. Leung GK, Lopes MB, Thorner MO, Vance ML, Laws ER Jr. Primary hypophysitis: a single-center experience in 16 cases. $\mathrm{J}$ Neurosurg. 2004;101:262-71.

3. Sumida M, Uozumi T, Mukada K, Arita K, Kurisu K, Eguchi K. Rathke cleft cysts: correlation of enhanced MR and surgical findings. AJNR. 1994;15:525-32.

4. Macaulay RJ. Ruptured Rathke's cleft cyst: a possible cause of Tolosa-Hunt syndrome. Clin Neuropathol. 1997;16:98-102.

5. Daikokuya H, Inoue Y, Nemoto Y, Tashiro T, Shakudo M, Ohata K. Rathke's cleft cyst associated with hypophysitis: MRI. Neuroradiology. 2000;42:532-4.

6. Sonnet E, Roudaut N, Mériot P, Besson G, Kerlan V. Hypophysitis associated with a ruptured Rathke's cleft cyst in a woman during pregnancy. J Endocrinol Invest. 2006;29:353-7.

7. Roncaroli F, Bacci A, Frank G, Calbucci F. Granulomatous hypophysitis caused by a ruptured intrasellar Rathke's cleft cyst: report of a case and review of the literature. Neurosurgery. 1998;43:146-9.

8. Wearne MJ, Barber PC, Johnson AP. Symptomatic Rathke's cleft cyst with hypophysitis. Br J Neurosurg. 1995;9:799-803.

9. Hama S, Arita K, Tominaga A, Yoshikawa M, Eguchi K, Sumida M, et al. Symptomatic Rathke's cleft cyst coexisting with central diabetes insipidus and hypophysitis: case report. Endocr $\mathrm{J}$. 1999;46:187-92.

10. Albini CH, MacGillivray MH, Fisher JE, Voorhess ML, Klein DM. Triad of hypopituitarism, granulomatous hypophysitis, and ruptured Rathke's cleft cyst. Neurosurgery. 1988;22:133-6.

11. Nishikawa T, Takahashi JA, Shimatsu A, Hashimoto N. Hypophysitis caused by Rathke's cleft cyst. Neurol Med Chir (Tokyo). 2007;47:136-9.

12. Murakami $M$, Nishioka $H$, Izawa $H$, Ikeda $Y$, Haraoka J. Cranulomatous hypophysitis associated with Rathke cleft cyst: a case report. Minim Invas Neurosurg. 2008;51:169-72. 